A 50-year-old man presented to the ear, nose, and throat casualty clinic with bleeding from the left external auditory meatus. He had undergone upper gastrointestinal endoscopy that morning without the use of a local anaesthetic throat spray or sedation. During the procedure, it was noted that the patient was gagging excessively, following which he experienced a searing pain in the left ear. On questioning, the patient stated that he had been suffering from a coryzal-type illness for the previous week and still had a blocked nose. Before this episode, he had not experienced any problems with his ears. On examination of the left ear, a small perforation was found on the anterior portion of the pars tensa. A diagnosis of tympanic perforation following upper gastrointestinal endoscopy was made, and the patient was treated with antibiotic ear drops for 1 week to prevent any secondary infection. No follow-up appointment was arranged [1].

The tympanic membrane perforation appears to have been due to positive pressure within the middle ear after the inadvertent performance of a forced Valsalva manoeuvre. There was a sudden over-admittance of air via the Eustachian tube to the tympanic cavity, causing lateral displacement of the tympanic membrane. In less severe cases, this would have caused hyperaemia, oedema, and ecchymosis. Valsalva manoeuvres are known to cause occasional perforations, but there is no previously documented evidence that a tympanic perforation has ever resulted from a gastrointestinal endoscopic procedure. The event was most likely due to a combination of factors, such as the lack of sedation and the incidental coryzal illness, leading to excessive gagging against a closed system.

\title{
Tympanic Perforation Following Upper Gastrointestinal Endoscopy
}

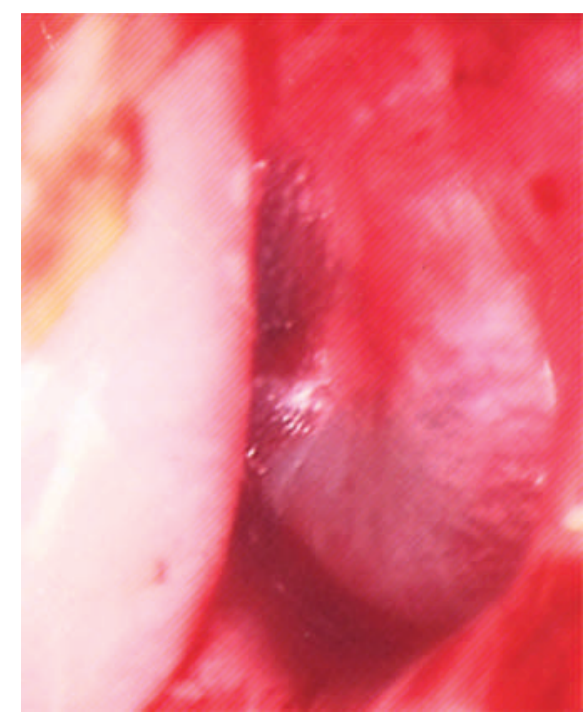

Figure 1 A small perforation is seen on the anterior portion of the pars tensa of the left ear. There is a small amount of bleeding, collecting both behind the tympanic membrane and in the external auditory canal.

\section{W. Mason}

Dept. of Ear, Nose, and Throat Medicine, Singleton Hospital, Swansea, Wales, United Kingdom.

\section{Reference}

\footnotetext{
${ }^{1}$ Deguine C, Pulec JL. Traumatic perforation: spontaneous healing after 3 months. Ear Nose Throat J 2003; 82: 544
}

\section{Corresponding Author}

L. W. Mason, M. B., B. Ch.

Dept. of Ear, Nose, and Throat Medicine, Singleton Hospital, Swansea 2 Pantbach Avenue Rhiwbina, Cardiff CF14 1UR

Wales

United Kingdom

Fax: $\quad$ +44-7947038874

E-mail: MasonLW@hotmail.com 